\title{
Advanced local voltage control through polynomial P-var functions
}

\author{
Heinrich, Carsten; Ziras, Charalampos; Bindner, Henrik W.
}

\section{Published in:}

Proceedings of 2019 IEEE Milan PowerTech

Link to article, DOI:

10.1109/PTC.2019.8810510

Publication date:

2019

Document Version

Peer reviewed version

Link back to DTU Orbit

Citation (APA):

Heinrich, C., Ziras, C., \& Bindner, H. W. (2019). Advanced local voltage control through polynomial P-var functions. In Proceedings of 2019 IEEE Milan PowerTech [8810510] IEEE. 2019 IEEE Milan PowerTech, PowerTech 2019 https://doi.org/10.1109/PTC.2019.8810510

\section{General rights}

Copyright and moral rights for the publications made accessible in the public portal are retained by the authors and/or other copyright owners and it is a condition of accessing publications that users recognise and abide by the legal requirements associated with these rights.

- Users may download and print one copy of any publication from the public portal for the purpose of private study or research.

- You may not further distribute the material or use it for any profit-making activity or commercial gain

- You may freely distribute the URL identifying the publication in the public portal

If you believe that this document breaches copyright please contact us providing details, and we will remove access to the work immediately and investigate your claim 


\title{
Advanced Local Voltage Control through Polynomial P-Var Functions
}

\author{
Carsten Heinrich, Charalampos Ziras and Henrik W. Bindner \\ Department of Electrical Engineering \\ Technical University of Denmark \\ Copenhagen, Denmark \\ \{cahei, chazi, hwbi\}@elektro.dtu.dk
}

\begin{abstract}
Reactive power control of inverter-based equipment, such as photovoltaic units or electric vehicles, is one way to address increasing voltage violations in distribution grids. Local droop control strategies have been rolled out in several European countries. In this work, we present an advanced method to define polynomial P-Var curves. A stochastic optimal power flow problem is solved, which co-optimizes both voltage deviations and distribution grid losses, and incorporates the polynomial coefficients as decision variables. The proposed method is applied to a real Danish low voltage feeder, leading to more robust solutions.
\end{abstract}

Index Terms-Optimal power flow, Power distribution, Power system planning, Reactive power control, Voltage control

\section{NOMENCLATURE}

Sets

I Set of buses

$T \quad$ Set of time steps

$T^{*} \quad$ Subset of time steps of cluster centers

\section{Variables}

$\eta \quad$ EV charging efficiency

$\lambda \quad$ Co-optimization trade off variable

$\pi_{t} \quad$ Cluster size of representative time step $t$

$a_{p}, b_{p}$, Polynomial P-Var curve parameters of PV unit $p$

$c_{p}, d_{p}$

$E_{\mathrm{EV}} \quad \mathrm{EV}$ battery capacity

$I_{i, j, t}^{\mathrm{m}} \quad$ Current magnitude flowing from node $i$ to node $j$ at time $t$

$I_{i, j}^{\text {rat }} \quad$ Current rating of equipment between node $i$ and node $j$

$P_{p}^{\mathrm{kWp}} \quad \mathrm{kW}$ peak of $\mathrm{PV}$ unit $p$

$P_{i, t}^{\mathrm{l}} \quad$ Active power consumption of households at node $\mathrm{i}$ and time $\mathrm{t}$

$P_{i, j, t} \quad$ Active power flowing from bus $i$ to bus $j$ at bus $i$ and time $\mathrm{t}$

$P_{p, t} \quad$ Active power output of PV unit $p$ at time $t$

$Q_{i, t}^{1} \quad$ Reactive power consumption at node $\mathrm{i}$ and time $\mathrm{t}$

$Q_{i, j, t}$ Reactive power flowing from bus $i$ to bus $j$ at bus $i$ time $t$

$Q_{p, t} \quad$ Reactive power output of PV unit $p$ at time $t$

$t_{\text {arrive }} \mathrm{EV}$ arrival time

$t_{\text {depart }} \quad \mathrm{EV}$ departure time

$V_{i, t}^{\mathrm{m}} \quad$ Voltage magnitude at bus $i$ and time $t$

$V^{\text {nom }}$ Nominal voltage magnitude

\section{INTRODUCTION}

The introduction of renewable generation is increasingly causing voltage problems in distribution grids, particularly towards the end of long low-voltage feeders. On the one hand, distributed generation can cause reversed power flows. On the other hand, electric vehicles (EVs) can significantly increase residential load during peak hours. These developments might eventually lead to both stronger voltage dips, as well as higher voltage spikes in the same distribution grid.

One way to counteract such voltage deviations is reactive power control (RPC), facilitated by inverter-based equipment. Germany has implemented a mandatory local RPC strategy for photovoltaic (PV) units. PV inverters must have the ability to generate power with power factors between 0.95 inductive and 0.95 capacitive for units with a peak power smaller than $13.8 \mathrm{~kW}$, and power factors between 0.9 inductive and 0.9 capacitive for units with a peak power greater than $13.8 \mathrm{~kW}$. The power factor thereby only depends on their active power output [1]. The same control strategy has been adapted by Denmark for PV units larger than $11 \mathrm{~kW}$ [2] and by Austria [3], whereas a similar strategy has been introduced in Italy [4]. Such local control strategies have the advantage of not requiring communication infrastructure. This implementation might help to prevent voltage limit violations, but it usually increases grid losses due to higher current magnitudes. In most distribution grids, voltages do not reach critical values, hence a fixed control curve for all grids is not beneficial [5]. Distribution system operators (DSOs) are allowed to define unit-specific P-var curves, however this possibility has so far barely been made use of.

Several methods to define alternative and more efficient local reactive power controllers have been proposed. The authors of [6] calculate piece-wise linear P-V-Var curves for each PV inverter, where the reactive power output depends on local voltage and active power generation. The curves are fitted to the results of an optimal power flow (OPF) calculation which minimizes losses and treats the reactive power output of PV inverters as the decision variables. Since voltages deviations are not represented in the objective function, the derived curves do not reduce voltage deviations, as long as these stay within the allowed bounds. Since higher voltages generally reduce grid losses, voltages above the nominal value 
are interpreted as being beneficial, with an ideal voltage magnitude of 1.1 p.u. at all nodes. If such curves were adopted, regular voltage violations would occur. In [7], the authors define piece-wise linear voltage-var curves in a similar fashion, but only minimize voltage deviations instead. This approach neglects grid losses, which are one of the main cost factors for DSOs and one of the key indicators for their performance. Both [6] and [7] include local voltage measurements into their local controller. The authors of [8] chose a co-optimization approach and thereby take into account that the minimization of voltage deviations through RPC and the minimization of grid losses represent conflicting objectives. P-Var curves are defined through a regression.

One has to note that [6], [7] and [8] first solve an optimization problem which defines an optimal reactive power set point for each time step and for each inverter. Once these set points have been calculated, the local control curve of each inverter is fitted to these values. The limitation of this approach lies in the fact that this fitted curve does not necessarily respect the grid constraints of the initial optimization problem.

The authors of [9], [10] define piece-wise linear P-Var curves and V-Var curves in combination with active power curtailment within the power flow optimization. However, using piece-wise linear functions introduces integer variables, which is computationally expensive, and therefore limits the authors to a total of ten PV-units.

This paper defines local RPC-control curves through the formulations of a stochastic optimization problem. P-Var curves are defined as $3^{\text {rd }}$ order polynomials, whose parameters are the decision variables of the OPF problem. Hence, instead of a two-step procedure, the problem is solved at once, allowing to take all grid constraints into account when defining the P-Var curves. Since polynomial P-Var curves are used, no integer variables are introduced and the problem can easily be applied to larger distribution grids, with a high number of PV-units. The problem is formulated as a co-optimization of grid losses and voltage deviations. The method is tested on a model of a real low voltage distribution feeder with a total of 131 households.

The paper is structured as follows: Section II introduces the method, proposed in this paper. Section III presents the case study, as well as the used models. Finally, Section IV concludes the paper.

\section{DERIVATION OF ADAPTED P-VAR CURVES}

\section{A. Distribution Grid Operation Constraints}

During distribution grid operation, there are two major factors DSOs have to consider. First, voltages at customer nodes have to stay within an allowed rage. The European norm EN 50160 defines this range as $\pm 10 \%$ of the nominal voltage $V^{\text {nom }}$ [11]. Even though local grid codes often require DSOs to keep voltages within an even smaller interval, the $10 \%$ limit will be considered throughout this paper, which is expressed as

$$
0.9 V^{\mathrm{nom}} \leq V_{i, t}^{\mathrm{m}} \leq 1.1 V^{\mathrm{nom}}
$$

$V_{i, t}^{\mathrm{m}}$ denotes the voltage magnitude at node $i$ during time step $t$. We denote the set of grid nodes as $I$ and the set of time steps in the simulation as $T$. PV power generation in the distribution network increases nodal voltages. Therefore, (1) limits the acceptable PV penetration in each distribution network.

The second important constraint is set through equipment ratings. All power equipment is designed for a maximal current $I^{\text {rat }}$. Exceeding the ratings for a long period of time reduces the expected lifetime or can destroy the equipment entirely. Therefore, DSOs have to ensure, that currents flowing through power lines and transformers stay within the allowed range, defined by

$$
-I_{i, j}^{\mathrm{rat}} \leq I_{i, j, t}^{\mathrm{m}} \leq I_{i, j}^{\mathrm{rat}}
$$

Here, $I_{i, j, t}^{\mathrm{m}}$ represents the magnitude of the current flowing through the equipment, connecting node $i$ and node $j$ at time $t$, whereas $I_{i, j}^{\text {rat }}$ denotes the corresponding rating. Ultimately, (2) also limits the acceptable PV penetration in a distribution feeder. Whether the voltage constraint or the thermal constraint is the limiting factor for the PV hosting capacity of a feeder is decided by the grid topology and the individual PV locations. Generally, urban feeders with shorter cables tend to be rather limited through the thermal constraint (2), whereas feeders in the countryside with longer cables tend to be limited through the voltage limit (1).

\section{B. Photovoltaic Inverter Operational Constraints}

All loads and generators in the power system have a power factor, defining the ratio of active and apparent power flowing through the equipment. A capacitive power factor increases voltage at the point of common coupling (PCC) through the generation of reactive power. An inductive power factors reduces voltage by absorbing reactive power. In inverter-based equipment this ratio can be adjusted, which again can be used to manipulate voltages in the grid. If the maximal acceptable $\mathrm{PV}$ penetration is defined through the voltage constraint, such reactive power control can be used to allow additional PV generation in the feeder.

Local reactive power curves are useful and comparably easy to implement. The default curve, used in Germany is depicted in Fig. 1. Denmark, Italy and Austria are using the same curve, however it is only being applied, when requested by the local DSO. Inverters, just like any other equipment in the electricity grid, are designed for a specific maximal current, which can be transformed into an apparent power rating $S^{\text {rat }}$. This limits both the active and reactive power output of PV inverters according to

$$
Q_{p, t}^{2}+P_{p, t}^{2} \leq S_{p}^{\mathrm{rat}^{2}}
$$

The index $p$ is used for the set of PV units. Due to the grid codes [1-4], it is nowadays common to oversize PV inverters, such that they can still provide sufficient reactive power while operating close to their peak power $P_{p}^{\mathrm{kWp}}$. Accordingly, PV units have the capability to generate or absorb reactive power $Q_{p, t}$ as follows:

$$
-Q_{p}^{\min } \leq Q_{p, t} \leq Q_{p}^{\min }
$$




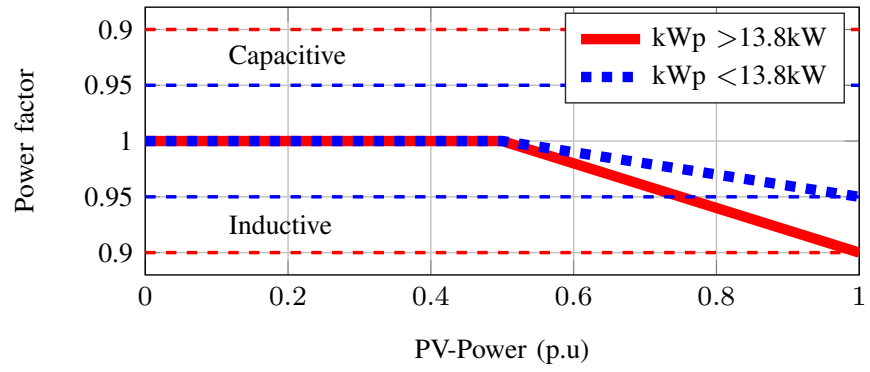

Figure 1. Characteristic curve and admissible range which is implemented by default into PV power inverters in Germany.

where

$$
Q_{p}^{\min }=\tan (\arccos (0.9)) P_{p}^{\mathrm{kWp}}
$$

Finally, PV inverters can be connected to a single phase or to all three phases. All grid codes limit the amount of asymmetric power injection to single phases (Germany 4.6kVA, Denmark, Austria and Italy $3.68 \mathrm{kVA}$ per phase). Beyond these limits, both active and reactive power has to be fed into the grid symmetrically on all phases.

\section{P-Var Curve Definition within the OPF}

We propose to derive P-Var curves based on historic smart meter data as well as historic solar irradiance data. It is impossible to solve the OPF for all time steps at once. In the following we present how a k-means clustering algorithm using the Euclidean norm is used to create representative scenarios of both load profiles and solar radiation [12]. Afterwards, the resulting scenarios are used to formulate a stochastic optimization problem. By solving this problem the integrated P-Var curves are defined.

1) Scenario Generation: The available data is split into a training set and a test set. The training set is used to create $\mathrm{P}$-Var curves, and afterwards their performance is evaluated on the test set. All the time steps of the training data are clustered into $k$ groups according to the following properties: total power consumption of all loads, total power consumption of all households on each of the branches, and the solar irradiance. The resulting amount of time steps assigned to each cluster is denoted as $\pi_{k}$. The time step with the smallest distance to the cluster center is chosen as the cluster's representative scenario. In addition to these $k$ time steps, two additional time steps are added to the scenarios: the time step where the solar irradiation is maximal, as well as the time step, when the total household and EV load is maximal. This way, the initial set of time steps $T$ is reduced to the subset $T^{*}$ containing only the $k+2$ representative time steps. In this work, five minute smart meter data and solar irradiance data from June and July 2016 have been used. June and July are used as a training and test set, respectively. The 8640 time steps are reduced to a total of 150 representative time steps, which can be seen in Fig. 2.

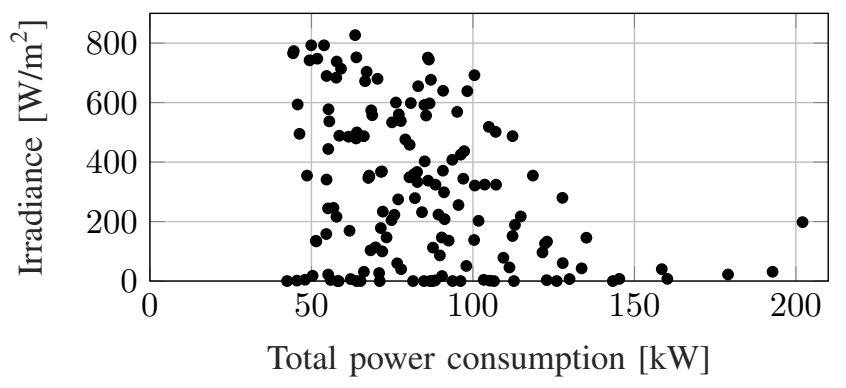

Figure 2. Representative time steps, chosen with the k-means algorithm.

2) Problem Formulation: The P-Var curves define the relation between active power generation $P_{p, t}$ and reactive power output $Q_{p, t}$ for each PV unit as

$$
Q_{p, t}=a_{p} P_{p, t}^{3}+b_{p} P_{p, t}^{2}+c_{p} P_{p, t}+d_{p}, \forall t, \forall p .
$$

These curves are derived by solving a stochastic OPF problem, which is formulated as a co-optimization of both grid losses and voltage deviations. We denote by $P_{i, j, t}$ the active power injected into the line connecting node $i$ and node $j$ at node $i$ and at time $t$. $\lambda$ denotes the trade off variable of the two objectives. $\pi_{t}$ weights the objective according to the cluster size. The objective function is formulated as

$$
\begin{aligned}
\min _{a_{p}, b_{p}, c_{p}, d_{p}} \lambda \sum_{i, j \in I, t \in T^{*}} \pi_{t} P_{i, j, t} \\
\quad+(1-\lambda) \sum_{i \in I, t \in T^{*}} \pi_{t}\left(V^{\mathrm{nom}}-V_{i, t}^{\mathrm{m}}\right)^{2} .
\end{aligned}
$$

The problem is solved for all 150 time steps resulting from the clustering at once. Each time step is weighed according to the cluster size $\pi_{t}$, representing the scenario's probability to occur. The first sum calculates the grid losses. By summing over all indices $i$ and $j \in I$, the power flowing from node $j$ to node $i$ is added to the power flowing from node $i$ to node $j$, resulting in the line losses. The second sum represents deviations from nominal voltage at all nodes. Deviations from nominal voltage are penalized quadratically, representing the fact that in the distribution grid small deviations are tolerable, while larger deviations should be avoided.

In addition, (1) to (5) are included as constraints. Finally, the usual $\mathrm{AC}$ power flow equations are also included, nodal active and reactive power balance as well as line flow equations.

\section{P-Var Definition based on Regression}

To compare the proposed method, we apply a two-step regression technique. First, the same OPF problem as in subsection II-C is solved for all time steps in the training set, with the only difference, that (6) is not included. The decisions variables are then the reactive power set points of the PV units $Q_{p, t}$. No clustering is necessary, since the problem can now be solved for all time steps individually. The objective function is then formulated as:

$$
\min _{Q_{p, t}} \lambda \sum_{i, j \in I, t \in T} P_{i, j, t}+(1-\lambda) \sum_{i \in I, t \in T}\left(V^{\mathrm{nom}}-V_{i, t}^{\mathrm{m}}\right)^{2} .
$$




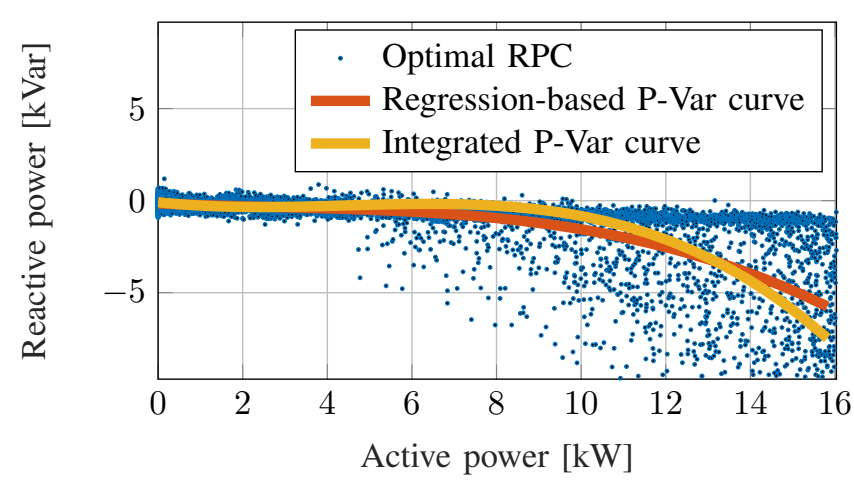

Figure 3. Q-P scatter plot of PV unit

Next, the polynomial coefficients are found by fitting a PVar curve. To do so, we solve the constrained linear least square problem, where the included constraint is (4). This problem is solved for each PV unit individually. Finally, to evaluate the performance of the two different P-Var curves, we apply them to the test set. As there are no free variables any more, this evaluation requires a simple load flow analysis. An example of the three optimization results for one PV inverter is depicted in Fig. 3. The yellow curve has been defined using the integrated approach from section II-C. Blue dots depict the individual time steps optimization result without (6), during the first step of the regression approach. Each dot represents the ideal reactive power set point of one time step. Based on this result, the red curve was fitted using the constraint least square method.

\section{CASE STUDY}

To test the proposed method, a real radial distribution feeder from the Danish island of Bornholm has been modeled, which is shown in Fig. 4. The grid connects a total of 131 residential houses. The red node marks the transformer station, where the system is connected to the higher voltage level through a $400 \mathrm{kVA}$ transformer. Yellow nodes indicate nodes to which PV-units were added blue nodes represent nodes with electric vehicles.

\section{A. Case study data and models}

1) Load and slack bus data: Loads are modeled as active and reactive power draws $\left(P_{i, t}^{1}, Q_{i, t}^{1}\right)$ at the PCC. The smart meter data for $P_{i, t}^{\mathrm{l}}$ and $Q_{i, t}^{\mathrm{l}}$ of 131 households with a resolution of five minutes are used for the simulation. Usually, several customers connect to the same node. In this case their load is aggregated. For the purpose of this simulation, it is assumed that the distribution grid is balanced. Slack bus voltages are modeled based on real five minute averaged voltage data from the SCADA-system. The used data which originates from the corresponding primary transformer station, has a mean voltage on the secondary side of $10.51 \mathrm{kV}$ and a standard deviation of $71.8 \mathrm{~V}$. These voltages are linearly mapped onto a distribution with the same shape, but with mean voltage of $10.1 \mathrm{~kW}$. To account for additional voltage

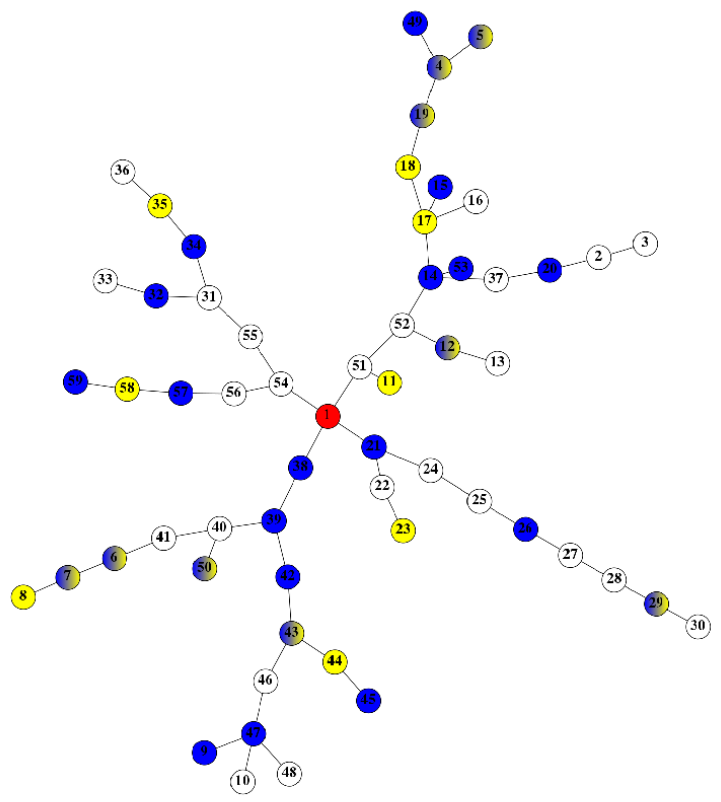

Figure 4. Sketch of the used $400 \mathrm{~V}$ distribution grid, the red node shows the secondary substation. PV units are located at yellow nodes, blue nodes depict EV-locations.

deviations through the $10 \mathrm{kV}$ grid a slightly higher standard deviation of $73.3 \mathrm{~V}$ is used for the slack bus.

2) Electric vehicles: To model a future load scenario of this distribution feeder, it is assumed that 39 of the 131 households own an electric vehicle. These cars charge such that they minimize the power acquisition cost on the wholesale market and reach a state of charge of $100 \%$ by the time they depart. Full knowledge of both spot prices [13] as well as arrival time $t_{\text {arrive }}$ and departure time $t_{\text {depart }}$ is assumed. Since households usually have a 16 Ampere fuse, cars charge with a maximal power of $3.68 \mathrm{~kW}$. Further, the modeled car batteries have a storage capacity of $24 \mathrm{kWh}$ and charge with an efficiency $\eta$. The probability distributions for $t_{\text {arrive }}, t_{\text {depart }}$ and the state of charge are taken from [14].

3) Photovoltaic units: Photovoltaic units are modeled as active power injections at the PCC. At 17 nodes PVs are placed into the grid. The injected power is proportional to the solar irradiance. The peak power of each PV unit $P_{p}^{\mathrm{kWp}}$ is fed into the grid, when the solar irradiance is $1 \mathrm{~kW} / \mathrm{m}^{2}$. Real solar irradiance data from the island of Bornholm is used for this purpose. A peak power $P_{p}^{\mathrm{kWp}}$ of $20 \mathrm{~kW}$ is used, which is rather large. This accounts for the fact, that at each node, several customers are connected.

The parameters for both PV units and EVs are summarized in Table I. The probability distribution for the state of charge at $t_{\text {arrive }}$ can be found in [14].

\section{B. Results}

The proposed method is applied to the months of June and July. In the considered distribution feeder, the highest voltages 


\begin{tabular}{cc|cc}
\hline \multicolumn{4}{c}{ Simulation } \\
Parameter & Value & Parameters & Value \\
\hline$t_{\text {arrive }}[\mathrm{h}]$ & $\mathcal{N}(19,10)$ & $t_{\text {depart }}[\mathrm{h}]$ & $\mathcal{N}(7.5,1)$ \\
$\eta^{+}(-)$ & $\mathbb{U}[0.85,0.9]$ & $P_{\text {charge, }}[\mathrm{kW}]$ & 3.68 \\
$E_{\mathrm{EV}}[\mathrm{kWh}]$ & 24 & $P_{\mathrm{p}}^{\mathrm{kWp}}[\mathrm{kW}]$ & 20 \\
\hline \multicolumn{4}{c}{ Table I }
\end{tabular}

SUMMARY OF SIMULATION PARAMETERS FOR PV AND EV MODEL.

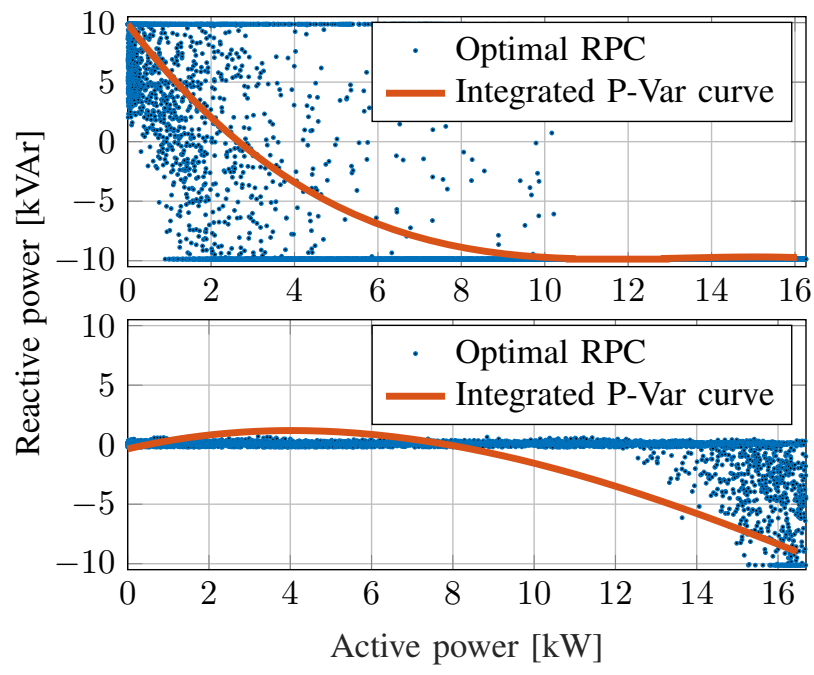

Figure 5. Integrated P-Var curve of PV unit at node 5. Top: minimization of voltage deviations, Bottom: minimization of losses.

occur at node five during times of high PV generation. Fig. 5 shows the calculated P-Var curve of the PV unit located at this bus. In the top graph, only voltage deviations are minimized $(\lambda=0)$. At low PV in-feed, the inverter uses a capacitive power factor to compensate for the voltage drop, caused by the residential loads. When PV generation exceeds the load, and power is flowing towards the transformer station, an inductive power factor reduces voltage.

The bottom graph shows the behavior of the same PV unit when only losses are minimized $(\lambda=1)$. For loss minimization, little reactive power is used by the PV inverters, since RPC usually leads to higher currents and therefore higher losses. The PV units in the grid only operate with a slight capacitive power factor to counteract the inductive loads and the transformer's inductiveness. During high power injections of the PV-units, reactive power is absorbed to lower the nodal voltage to the allowed range.

In this case study, four different control strategies are considered:

- Strategy 1: No RPC,

- Strategy 2: Ideal RPC with optimal reactive power set point,

- Strategy 3: Local RPC with P-Var curve based on regression method,

- Strategy 4: Local RPC with P-Var curve based on integrated calculation.

Strategies 1 and 2 are included as benchmarks, to illustrate

\begin{tabular}{|c|c|c|c|c|}
\hline \multicolumn{5}{|c|}{ Results $-\lambda=1$} \\
\hline Parameter & Str. 1 & Str. 2 & Str. 3 & Str. 4 \\
\hline Max. nodal voltage (p.u.) & 1.122 & 1.100 & 1.115 & 1.096 \\
\hline Min. nodal voltage (p.u.) & 0.976 & 0.973 & 0.965 & 0.970 \\
\hline time share with violations $(\%)$ & 3.9 & 0 & 2.25 & 0 \\
\hline Max. line loading (\%) & 88.6 & 88.6 & 89.2 & 99.6 \\
\hline Losses $(\%)$ & 2.18 & 2.18 & 2.20 & 2.49 \\
\hline Objective & 136.0 & 136.2 & 137.3 & 155.9 \\
\hline \multicolumn{5}{|c|}{ Results $-\lambda=0$} \\
\hline Max. nodal voltage (p.u.) & 1.122 & 1.088 & 1.092 & 1.092 \\
\hline Min. nodal voltage (p.u.) & 0.976 & 0.982 & 0.969 & 0.971 \\
\hline time share with violations $(\%)$ & 3.9 & 0 & 0 & 0 \\
\hline Max. line loading (\%) & 88.6 & 96.9 & 100.6 & 97.6 \\
\hline Losses $(\%)$ & 2.18 & 6.37 & 5.22 & 5.85 \\
\hline Objective & 204.1 & 35.7 & 70.3 & 65.9 \\
\hline \multicolumn{5}{|c|}{ Results $-\lambda=0.4$} \\
\hline Max. nodal voltage (p.u.) & 1.122 & 1.093 & 1.094 & 1.0944 \\
\hline Min. nodal voltage (p.u.) & 0.976 & 0.980 & 0.970 & 0.972 \\
\hline time share with violations $(\%)$ & 3.9 & 0 & 0 & 0 \\
\hline Max. line loading (\%) & 88.6 & 96.9 & 100.5 & 98.4 \\
\hline Losses $(\%)$ & 2.18 & 3.92 & 3.61 & 3.55 \\
\hline Objective & 127.6 & 52.7 & 70.4 & 66.0 \\
\hline
\end{tabular}

Table II

SUMMARY OF RESULTS FOR DIFFERENT $\lambda$

what the worst case scenario is (strategy 1) and what the best possible performance could be (strategy 2). Note that $4^{\text {th }}$-order polynomials have been tested as well, but it was found that these did not improve the performance on the test set, indicating over-fitting. Table II summarizes the results of these four control strategies for three different trade off values, $\lambda=1, \lambda=0.4$ and $\lambda=0$.

The first column is independent from $\lambda$, since it represents the case without RPC. This case leads to significant voltage violations in the distribution grid. $3.9 \%$ of the time the grid constraints are not respected in this scenario, corresponding to 56 minutes per day. When $\lambda=1$, only losses are minimized and nodal voltages are only represented through constraints. Using an ideal central controller, with full knowledge of the grid state (strategy 2), manages to contain all voltages in the allowed band, without any significant increase in grid losses. Using the regression-based approach (strategy 3) still leads to voltage violations $2.25 \%$ of the time, corresponding to 32 minutes per day. The integrated P-Var approach manages to respect all voltage constraints. To accomplish this, a larger share of reactive power is absorbed by the inverters, which results in an increase of losses of $0.3 \%$. Fig. 6 shows a logarithmic histogram of the nodal voltages throughout the distribution grid for these four different control strategies.

When only voltage deviations are minimized, all RPC controllers are able to contain the voltages. Since grid losses are not taken into account, this increases line loadings and therefore losses. Power ratings are only represented through constraints. When a regression-based P-Var curve is applied (strategy 3), these constraints can not be taken into account and the power line connecting the nodes 14 and 17 is at times overloaded. When the integrated P-Var curve is used, the lines are also heavily loaded, but all grid constraints are respected.

Finally, we analyze a trade-off case, where both objectives are taken into account with a similar weight. $\lambda$ is therefore 


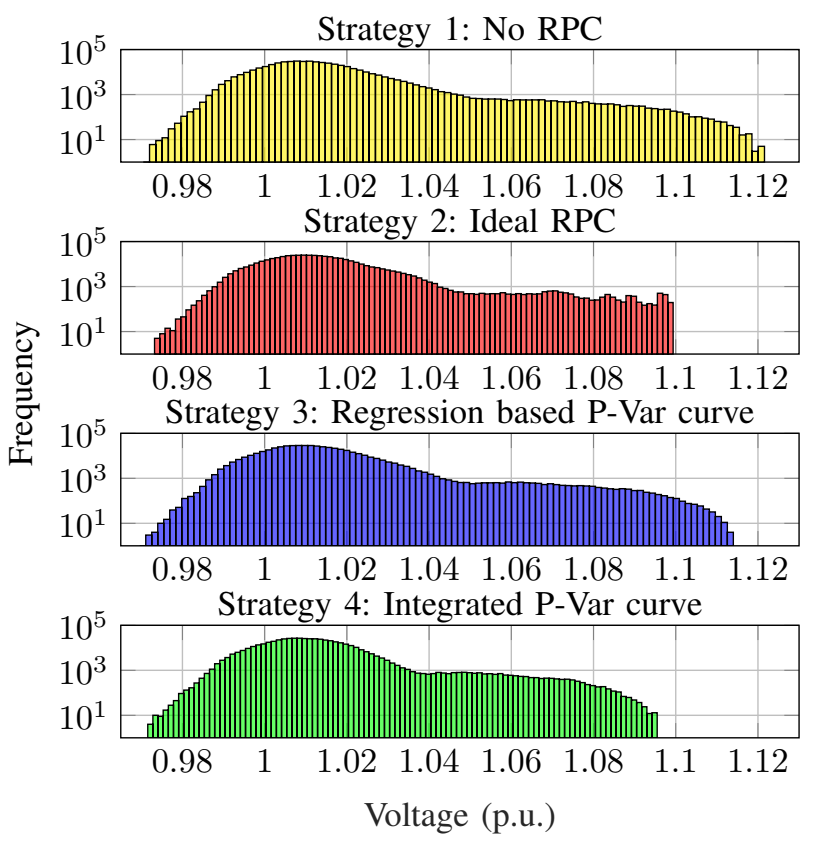

Figure 6. Logarithmic histogram of nodal voltages in the distribution grid for the four control strategies $(\lambda=0)$.

chosen based on the previous simulations objective results as $\lambda=0.4$. In this case, again all RPC controllers are able to respect the voltage constraint of the distribution gird. Regression based P-Var curves fail to fully respect the thermal constraint of the network. However, the application of integrated P-Var curves respect all constraints of the grid, while at the same time performing better in terms of grid losses and operator objective.

\section{CONCLUSION}

The continuing increase of distributed renewable generation in low voltage networks requires to control voltages at the customer side. Local RPC curves, where inverter-based equipment adjusts the reactive power output according to locally measured quantities, have already become part of grid codes in Germany, Denmark, Austria and Italy. Adjustments of such $\mathrm{P}-$ Var curves can further reduce grid losses, as well as voltage deviations.

This work presents a method to incorporate the derivation of P-Var curves into an optimal power flow calculation. By formulating the OPF as a co-optimization of grid losses and voltage deviations, the shape of these P-Var curves can be chosen according to the priorities of the local DSO. The derivation of curves through a regression technique is compared with the proposed integrated approach on a Danish lowvoltage distribution feeder. We show that when regression is applied, regular voltage or thermal constraint violations can occur, as not all grid constraints can be taken into account. Our calculation method is able to limit such constraint violations, since P-Var curves are defined within the optimal power flow calculation. Since polynomial curves, rather than piece-wise linear curves, are used, computational complexity is low and the problem scales well because of the employed polynomial curves instead of binary variables.

Future work will apply the proposed methodology to unbalanced grids. The PV units, which were considered in this study, are required to feed active and reactive power symmetrically into the different phases. However, the asymmetrical residential loads and $\mathrm{EV}$ charging are expected to have an influence on the ideal shape of P-Var curves. The result of such a study might indicate which load and grid topology information has to be taken into account when deriving advanced P-Var curves.

\section{ACKNOWLEDGMENT}

The authors would like to acknowledge the financial support of the EUDP project, EcoGrid 2.0, No 64015-002.

\section{REFERENCES}

[1] "VDE-AR-N 4105: Power generation systems connected to the lowvoltage distribution network, Technical minimum requirements for the connection to and parallel operation with low-voltage distribution networks, english translation," Verband der Elektrotechnik Elektronik Informationstechnik e.V., Tech. Rep., 2011.

[2] "Technical regulation 3.2. 2 for PV power plants with a power output above 11 kW," Energinet, DK, Tech. Rep., 2015.

[3] "Technische und organisatorische Regeln für Betreiber und Benutzer von Netzen, Hauptabschnitt D4: Parallelbetrieb von Erzeugungsanlagen mit Verteilernetzen (Technical and organizational rules for operators and users of grids, Part D4 Parallel operation of generation facilities with distribution networks), Version 2.3," 2016.

[4] "Reference Technical Rules for the Connection of Active and Passive Users to the LV Electrical Utilities," Comitato Elettrotecnico Italiano, Tech. Rep., 2012.

[5] C. Heinrich, P. Fortenbacher, A. Fuchs, and G. Andersson, "PVintegration strategies for low voltage networks," in 2016 IEEE International Energy Conference (ENERGYCON). IEEE, 2016.

[6] S. Karagiannopoulos, P. Aristidou, and G. Hug, "Hybrid approach for planning and operating active distribution grids," IET Generation, Transmission \& Distribution, vol. 11, no. 3, pp. 685-695, 2017.

[7] A. O'Connell and A. Keane, "Volt-var curves for photovoltaic inverters in distribution systems," IET Generation, Transmission \& Distribution, vol. 11, no. 3, pp. 730-739, 2017.

[8] O. Sondermeijer, R. Dobbe, D. Arnold, C. Tomlin, and T. Keviczky, "Regression-based inverter control for decentralized optimal power flow and voltage regulation," in Power \& Energy Society General Meeting. IEEE, 2016

[9] S. Weckx, C. Gonzalez, and J. Driesen, "Combined central and local active and reactive power control of PV inverters," IEEE Transactions on Sustainable Energy, vol. 5, no. 3, pp. 776-784, 2014.

[10] S. Weckx and J. Driesen, "Load balancing with EV chargers and PV inverters in unbalanced distribution grids," IEEE Transactions on Sustainable Energy, vol. 6, no. 2, pp. 635-643, 2015.

[11] "EN 50160: Voltage Characteristics of electricity supplied by public distribution networks," European Committee for Electrotechnical Standardization, Standard, 2010.

[12] S. Lloyd, "Least squares quantization in pcm," IEEE transactions on information theory, vol. 28, no. 2, pp. 129-137, 1982

[13] Nord Pool AS, "Historical Market Data Nord Pool," https://www.nordpoolgroup.com/Market-data1/Dayahead/AreaPrices/DK/Hourly/?view=table, online; accessed 12-December-2018.

[14] F. Rassaei, W.-S. Soh, and K.-C. Chua, "Demand response for residential electric vehicles with random usage patterns in smart grids," IEEE Transactions on Sustainable Energy, vol. 6, no. 4, pp. 1367-1376, 2015. 